\title{
Mit Berufserfahrung zum anerkannten MPA-Abschluss
}

\author{
Bettina Wöhlera, Verena Gantner \\ ${ }^{\text {a }}$ Fachfrau Validierung, biz Oerlikon, Kanton Zürich Bildungsdirektion; ${ }^{b}$ Dr. med., Fachärztin für Allgemeine Innere Medizin, Mitglied FMH
}

Das Validierungsverfahren zur Medizinischen Praxisassistentin bzw. zum Medizinischen Praxisassistenten EFZ ermöglicht, beruflich erworbene Fähigkeiten und Kenntnisse anerkennen zu lassen. Es richtet sich an Erwachsene, die keinen formalen Berufsabschluss absolviert haben oder als Quereinsteigende im Berufsfeld medizinische Praxisassistenz (MPA) arbeiten und nun den fehlenden Abschluss erwerben möchten.

Der Kanton Zürich bietet seit 2005 die Validierung von Bildungsleistungen an, seit 2010 ist das Validierungsverfahren vom Staatssekretariat für Bildung, Forschung und Innovation SBFI bewilligt. Ab erstem Quartal 2017 wird es nun auch möglich sein, über den Weg der Validierung ein eidgenössisches Fähigkeitszeugnis (EFZ) als Medizinische Praxisassistentin bzw. Medizinischer Praxisassistent (MPA) zu erwerben. Das Validierungsverfahren ist in fünf Phasen unterteilt:

\section{Phase 1 - Information und Selbst- einschätzung}

Die Teilnehmenden besuchen den obligatorischen Informationsanlass I und erhalten alle notwendigen Informationen zum Validierungsverfahren. Mittels eines Selbstchecks, eines diagnostischen Mittels als Entscheidungshilfe, können die Teilnehmenden einschätzen, ob das Validierungsverfahren für sie der richtige Weg zum EFZ ist. Der obligatorische Informationsanlass II führt danach vertieft ins Verfahren ein.

\section{Ärztinnen und Ärzte gesucht!}

Für das Validierungsverfahren suchen wir Ärztinnen und Ärzte, welche die Dossiers beurteilen. Erfahrene MPA beurteilen die Dossiers gemäss den erarbeiteten Richtlinien mit. Wir erachten eine Mitsprache von ärztlicher Seite bei der Beurteilung des gesamten Bildungsleistungspaketes einer Kandidatin als zentral: Die Beteiligung der Arbeitgeber bei der Beurteilung der Dossiers sichert die Akzeptanz der Abschlüsse auf dem Arbeitsmarkt. Deshalb möchten wir Kolleginnen und Kollegen, welche gegenüber der Durchlässigkeit in unserem Bildungssystem aufgeschlossen sind, als Experten gewinnen. Zur Einführung in die Dossierbeurteilung findet für alle Interessierten ein zweitägiger Kurs statt. Weitere Auskünfte erteilen wir Ihnen gerne über mpa[at]fmh.ch.

\section{Phase 2 - Handlungskompetenzen}

Die Teilnehmenden bereiten den Zulassungsantrag vor. Voraussetzung für das Validierungsverfahren sind fünf Jahre Berufserfahrung, wovon drei Jahre im Bereich der medizinischen Praxisassistenz. Gleichzeitig kann die anrechenbare Vorbildung angegeben werden. Anrechenbar sind berufliche und allgemeinbildende Handlungskompetenzen aus definierten Vorbildungen.

Die fehlenden Handlungskompetenzen können im Dossier anschliessend wie folgt nachgewiesen werden:

- Im schriftlichen Erfahrungsbericht beschreiben, begründen und reflektieren die Teilnehmenden ihr konkretes Vorgehen im beruflichen Alltag.

- Im mündlichen Erfahrungsbericht erzählen bzw. demonstrieren die Teilnehmenden mit individuellen Hilfsmitteln ihre beruflichen Handlungskompetenzen vor zwei Experten.

- Beim Praxisbesuch geht ein Experte an den Arbeitsplatz der Teilnehmenden und beobachtet sie über einen definierten Zeitraum bei der Arbeit.

Haben die Teilnehmenden ihre Handlungskompetenzen nachgewiesen und liegt die Zulassung vor, können sie ihr Dossier einreichen.

\section{Phase 3 - Beurteilung durch Experten}

In der Regel beurteilen zwei Experten das Dossier. Ist die Allgemeinbildung auch Teil des Dossiers, dann erfolgt die Beurteilung durch drei Experten. Diese laden zu einem Beurteilungsgespräch ein, in dem offene Fragen geklärt werden und das Dossier verifiziert wird. Das Gespräch dauert 45 Minuten, mit Allgemeinbildung jeweils 60 Minuten. Die Experten erstellen einen Be- 
urteilungsbericht, den diese an die Prüfungskommission senden.

\section{Phase 4 - Überprüfung durch Kommission}

Die Prüfungskommission überprüft den Beurteilungsbericht und stellt eine Lernleistungsbestätigung aus. Damit wissen die Teilnehmenden, welche beruflichen und allgemeinbildenden Handlungskompetenzen angerechnet werden und welche noch zusätzlich zu erwerben sind.

Fehlende berufliche und allgemeinbildende Kompetenzen können an der Schule Juventus Woodtli in Zürich nachgeholt und mit einer mündlichen, schriftlichen und/oder praktischen Prüfung nachgewiesen werden.

rrespondenz:

FMH Verbindung der

Schweizer Ärztinnen

und Ärzte

MPA-Sekretariat

Elfenstrasse 18

Postfach 300

CH-3000 Bern 15

Tel. 0313591111

mpa[at]fmh.ch

\section{Phase 5 - EFZ-Antrag}

Sind alle notwendigen Belege vorhanden, kann das EFZ beantragt werden. Die Prüfungskommission evaluiert, ob die Bestehensregeln für den Berufsabschluss erfüllt sind, und stellt das EFZ aus.

\section{Weitere Information, nützliches Tool und Coaching}

Das Validierungsverfahren zur Medizinischen Praxisassistentin bzw. zum Medizinischen Praxisassistenten ist eine gute Möglichkeit, berufsbegleitend einen $\mathrm{Ab}$ schluss zu erwerben. Es wird im ersten Quartal 2017 starten. Nähere Informationen finden Sie ab diesem Zeitpunkt unter www.validierung.zh.ch sowie unter www.mpaschweiz.ch $\rightarrow$ Validierung von Bildungsleistungen.

Zur Abwicklung des Validierungsverfahrens hat der Kanton Zürich ein webbasiertes Validierungstool entwickelt. Es führt die Teilnehmenden durch die einzelnen Phasen, unterstützt sie bei der Zulassung und Dossiererstellung. Das Validierungstool ermöglicht ein zeitlich und örtlich unabhängiges Arbeiten am Dossier.

Die Fachstelle Validierung des biz Oerlikon begleitet auf Wunsch die Teilnehmenden während der Dossiererstellung. Im Einzelcoaching können individuelle Fragen geklärt, Kompetenznachweise geplant, vorbereitet und besprochen werden. 